\title{
Term Neonate with Atypical Hypoxic-Ischemic Encephalopathy Presentation: A Case Report
}

\author{
Nick Townley, MD ${ }^{1} \quad$ Emily McNellis, $M^{1} \quad$ Venkatesh Sampath, MD \\ ${ }^{1}$ Division of Neonatology, Department of Pediatrics, Children's Mercy \\ Hospital, Kansas City, Missouri \\ Am J Perinatol Rep 2017;7:e171-e173. \\ Address for correspondence Nick Townley, MD, Division of \\ Neonatology, Department of Pediatrics, Children's Mercy Hospital, \\ 2401 Gillham Road, Kansas City, MO 64108 \\ (e-mail: ntownley@cmh.edu).
}

\author{
Abstract \\ Keywords \\ - Hypoxic-ischemic \\ encephalopathy \\ - umbilical vein \\ thrombosis \\ - status epilepticus
}

We describe a case of atypical hypoxic-ischemic encephalopathy (HIE) in a neonate following a normal pregnancy and delivery who was found to have an umbilical vein thrombosis. The infant arrived to our center with continuous bicycling movement of her lower extremities. She had a continuous electroencephalogram that showed burst suppression and magnetic resonance imaging of the brain showed diffusely abnormal cerebral cortical/subcortical diffusion restriction which may be secondary hypoxicischemic injury. Interestingly, a pathology report noted a focal umbilical vein thrombosis appearing to have compressed an umbilical artery with associated arterial dissection and hematoma. Our case illustrates how umbilical venous or arterial thrombosis may be associated with HIE and refractory seizures.
Hypoxic-ischemic encephalopathy (HIE) occurs in 2.5 per 1,000 term births making it a common admission diagnosis in tertiary neonatal intensive care units (NICUs). ${ }^{1}$ Neonates with HIE can have a history of birth trauma, birth difficulty, or depression after delivery but not in all cases. ${ }^{2}$ We describe a case of atypical HIE in a neonate following a reportedly normal pregnancy and delivery who was subsequently found to have an umbilical vein thrombosis.

\section{Case Presentation}

A female infant was born by spontaneous vaginal delivery at 41 weeks of gestation to a 25 -year-old gravida 2 , para 2 mother. The mother was obese but otherwise healthy, had received routine prenatal care, and had no history of previous miscarriages. Her first pregnancy was unremarkable and the family had a healthy 3-year-old child. Delivery history was significant for premature rupture of membranes for 22 hours. There were no concerns on prenatal ultrasounds nor evidence of fetal instability during the delivery process. There was no family history of thrombosis or clotting disorders. The infant's birth weight was $3.4 \mathrm{~kg}$, head circumference $34.5 \mathrm{~cm}$, and length $49.5 \mathrm{~cm}$, all appropriate for gestational age. A nuchal cord was wrapped two times, that was easily reduced, and the mother had a temperature of $39.2^{\circ} \mathrm{C}$ at delivery. Apgar scores were 2 at 1 minute, 6 at 10 minutes, and 8 at 10 minutes of life. Poor scores were due to inadequate respiratory effort, and the infant was tracheally intubated in the delivery room. The infant's heart rate did not drop below 60 and was above 100 with positive pressure ventilation. Cord gases were unremarkable (arterial pH 7.24/base excess [BE] - 6 and venous pH 7.32/ $\mathrm{BE}-8$ ) and the initial arterial blood gas after intubation was $7.24 / 18 / 104 /-16$ showing partially compensated metabolic acidosis.

The infant was on low conventional ventilation settings and was extubated after being moved to the NICU. Chest film was unremarkable. Hemodynamically, the infant was stable for the first 8 hours of life. After 8 hours of life, her family and clinicians were concerned about posturing movement, apnea, and desaturations. The infant was transferred to a regional referral NICU after being given a loading dose of phenobarbital. The physical exam after arriving to the referral center was notable for increased upper extremity tone, static extension of both upper extremities, and a continuous bicycling movement of her lower extremities. The exam at 10 hours of life was consistent with encephalopathy and status epilepticus. She was intubated to support her airway and an umbilical venous line was placed. received

November 29, 2016 accepted after revision June 30, 2017
DOI https://doi.org/

10.1055/s-0037-1605372. ISSN 2157-6998.
Copyright $\odot 2017$ by Thieme Medical Publishers, Inc., 333 Seventh Avenue, New York, NY 10001, USA. Tel: +1(212) 584-4662.
License terms

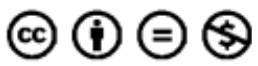




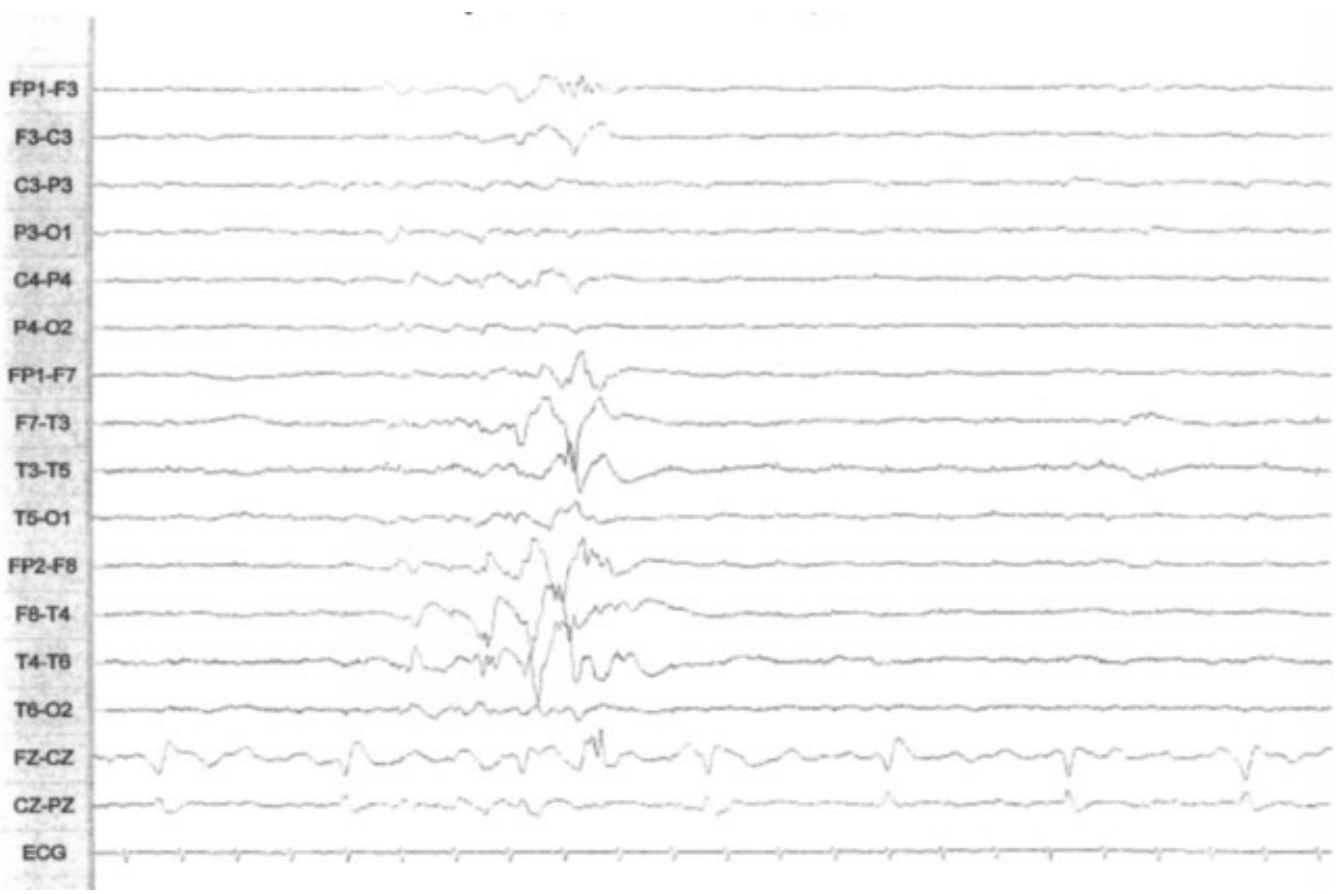

Fig. 1 Electroencephalogram with burst suppression.

Clinical and amplitude-integrated electroencephalogram (aEEG) monitored seizures were refractory and required escalation of seizure medication until a midazolam-induced coma was initiated and seizures resolved. She remained on low ventilator settings. She was monitored by continuous EEG that showed burst suppression (-Fig. 1). Magnetic resonance imaging (MRI) of the brain at 22 hours of life showed diffusely abnormal cerebral cortical/subcortical diffusion restriction which may be secondary hypoxic-ischemic injury (-Fig. 2). As this infant did not appear symptomatic until 8 hours of life, and admission to the tertiary center did not occur until 10 hours of life, therapeutic cooling was not pursued.

Infectious diseases or inborn errors of metabolism, while rare, can present as an atypical case of HIE. In this case, cerebral spinal fluid evaluation revealed unremarkable neurotransmitter, amino acid and cell count profiles, negative herpes simplex virus types 1 and 2, and bacterial and common viral cultures. Urine analysis was negative for organic acids and ketones, and serum revealed normal levels for ammonia, acylcarnitine, pyruvate, sulfocysteine, and amino acids profile. On day 7 of life, a pathology report noted a focal umbilical vein thrombosis appearing to have compressed an umbilical artery with associated arterial dissection and hematoma. Normal cord length, without other cord or placental anomalies was noted and development of the event was suggested to have been sudden per pathologist. Given the atypical presentation of HIE, this suggested the etiology of the patient's presentation. After a 2-day midazolam wean, the infant was discharged to home hospice on day 9 of life continuing maintenance phenobarbital. Her mother was to undergo a screening evaluation for a possible inherited thrombophilia disorder.

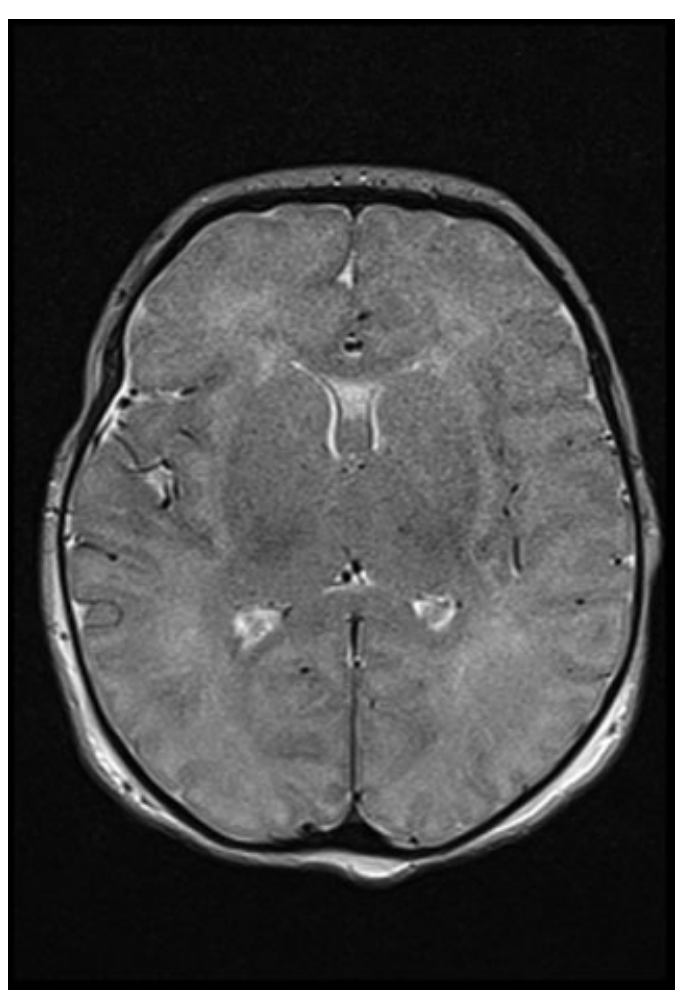

Fig. 2 Magnetic resonance imaging of the brain showing diffusely abnormal restricted diffusion throughout the cerebral cortex involving the bilateral frontal, bilateral parietal, left greater than right occipital, and temporal regions, predominantly in the cortex and subcortical white matter. The edema in this image shows the event was a global insult. 


\section{Discussion}

HIE can be traceable to an antenatal or perinatal event such as abruption or cephalopelvic disproportion. ${ }^{2}$ Atypical presentations have been associated with inborn errors of metabolism (urea cycle disorders, organic and amino acid disorders, glutaric aciduria) or meningitis. All were screened for and ruled out in our patient. Umbilical cord thrombosis has been reported and is correlated with additional umbilical cord abnormalities. ${ }^{3,4}$ Its incidence is estimated to occur in 1 of 1,300 deliveries, ${ }^{5}$ and there are a few reports on cord vessel dissection and thrombosis resulting in $\mathrm{HIE},{ }^{6,7}$ stillbirth, or complicated NICU course. ${ }^{8}$ The case presented broadens the potential etiologies of HIE and underscores the importance of pathological evaluation of the placenta and umbilical cord in these cases.

Reported risk factors for thrombosis in the fetus or products of conception include maternal diabetes, ${ }^{3}$ nuchal cord, ${ }^{4,6}$ and maternal obesity with the latter two risk factors present in our patient. The initial thrombotic event may then have been associated with vascular congestion, hypovolemia, hypoxia, and anemia, thus leading to neurological injury that later presented with seizures. In one animal study, intermittent partial cord occlusions in fetal lambs led to death in one-third of the subjects and high rates of status epilepticus and EEG suppression in the survivors, similar to our case. ${ }^{9}$

The patient's perinatal history, Apgar scores, initial blood gas, and reassuring cord gases did not guarantee an acute perinatal event making clinical association necessary in determining the cause of intrapartum hypoxia. As the timing of the neurological injury could not be elucidated by MRI, the initial injury may have been remote allowing some recovery prior to delivery or immediately preceding delivery, not allowing acidosis to develop. The normal cord gases could suggest such recovery via recanalization and re-establishment of sufficient perfusion.

\section{Conclusion}

HIE is a common presentation to referral NICUs that can be linked to a history of a traumatic event at birth and less commonly with metabolic or infectious causes. Our case is unique because the presentation of HIE and refractory seizures is associated with umbilical venous thrombosis. This presentation is atypical for HIE but important for clinicians to consider during a neurological insult work-up. This case highlights that while history and work-up are important, pathological evaluation for placental and umbilical cord abnormalities may be just as vital in the search of an etiology for atypical presentations of HIE, thus broadening the differential in a common NICU diagnosis.

\section{References}

1 Gillam-Krakauer M, Carter BS. Neonatal hypoxia and seizures. Pediatr Rev 2012;33(09):387-396, quiz 397

2 Martinez-Biarge M, Diez-Sebastian J, Wusthoff CJ, Mercuri E, Cowan FM. Antepartum and intrapartum factors preceding neonatal hypoxic-ischemic encephalopathy. Pediatrics 2013;132(04):e952-e959

3 Parast MM, Crum CP, Boyd TK. Placental histologic criteria for umbilical blood flow restriction in unexplained stillbirth. Hum Pathol 2008;39(06):948-953

4 Redline RW. Clinical and pathological umbilical cord abnormalities in fetal thrombotic vasculopathy. Hum Pathol 2004;35(12):1494-1498

5 Heifetz SA. Thrombosis of the umbilical cord: analysis of 52 cases and literature review. Pediatr Pathol 1988;8(01):37-54

6 Chan JS, Baergen RN. Gross umbilical cord complications are associated with placental lesions of circulatory stasis and fetal hypoxia. Pediatr Dev Pathol 2012;15(06):487-494

7 Baergen RN. Cord abnormalities, structural lesions, and cord “accidents". Semin Diagn Pathol 2007;24(01):23-32

8 Shilling C, Walsh C, Downey P, Mooney E. Umbilical artery thrombosis is a rare but clinically important finding: a series of 7 cases with clinical outcomes. Pediatr Dev Pathol 2014;17(02):89-93

9 Drury PP, Davidson JO, van den Heuij LG, et al. Status epilepticus after prolonged umbilical cord occlusion is associated with greater neural injury in [corrected] fetal sheep at term-equivalent. PLoS One 2014;9(05):e96530 